\title{
Editorial: Plant Glycobiology - A Sweet World of Glycans, Glycoproteins, Glycolipids, and Carbohydrate-Binding Proteins
}

\author{
Georg J. Seifert ${ }^{1}$, Richard Strasser ${ }^{1}$ and Els J. M. Van Damme ${ }^{2 *}$ \\ ${ }^{1}$ Department of Applied Genetics and Cell Biology, University of Natural Resources and Life Sciences, Vienna, Austria, \\ ${ }^{2}$ Department of Biotechnology, Ghent University, Ghent, Belgium
}

Keywords: protein glycosylation, post-translational modification, lectin, carbohydrate, cell wall polysaccharide

Editorial on the Research Topic

Plant Glycobiology - A Sweet World of Glycans, Glycoproteins, Glycolipids, and Carbohydrate-Binding Proteins

\section{INTRODUCTION}

Plants synthesize a wide variety of glycan structures which play essential roles during plant development and contribute to many diverse processes. These glycans function as structural components in the plant cell wall, assist in the folding and secretory trafficking of nascent proteins, act as signaling molecules in stress and plant defense responses, or serve within the energy metabolism of a plant (Strasser, 2016). Studying the biological roles of plant glycans enables a better understanding of plant growth and development under various environmental conditions in order to protect plants and exploit them for agriculture, forestry, or biotechnological products (De Coninck et al., 2021).

This Research Topic consists of 32 papers, including 10 review papers, 21 original research articles and 1 perspective article, and provides an overview of the latest research, methodologies and applications related to the fascinating world of plant glycobiology.

orrespondenct

Els J. M. Van Damme ElsJM.VanDamme@ugent.be

Specialty section: This article was submitted to Plant Proteomics and Protein

Structural Biology, a section of the journal

Frontiers in Plant Science

Received: 02 August 2021 Accepted: 09 August 2021 Published: 03 September 2021

Citation:

Seifert GJ, Strasser $R$ and Van Damme EJM (2021) Editorial: Plant

Glycobiology - A Sweet World of Glycans, Glycoproteins, Glycolipids, and Carbohydrate-Binding Proteins.

Front. Plant Sci. 12:751923

doi: $10.3389 / \mathrm{fp} / \mathrm{s} .2021 .751923$

\section{N-GLYCANS AND THEIR ROLE IN PLANT DEVELOPMENT}

$\mathrm{N}$-glycosylation is an abundant posttranslational modification of proteins entering the secretory pathway in all eukaryotes. It is well-established that the attached $N$-glycans have an impact on protein folding and quality control processes. The function of specific $N$-linked glycan modifications is, however, still obscure in plants (Nagashima et al., 2018). In this Research Topic several original research papers and reviews report new findings and highlight important aspects of specific $N$-glycan modifications.

The review by Zhang et al. highlights the role of a distinct oligomannosidic $N$-glycan as a signal for termination of quality control processes and initiation of ER-associated degradation (ERAD). The authors provide detailed insights into conserved and unique features of mammalian, yeast and plant $\alpha$-mannosidases involved in ERAD of misfolded glycoproteins. While a coherent picture is emerging for the early steps of the ERAD pathway, later steps that involve retrotranslocation of the misfolded glycoprotein to the cytosol and clearance are still poorly characterized. Prior to degradation by the proteasome, ERAD substrates are predicted to undergo deglycosylation by the sequential action of two cytosolic enzymes, peptide: $N$-glycanase and 
endo- $\beta-N$-acetylglucosaminidase, resulting in the release of oligomannosidic free $N$-glycans from the misfolded glycoproteins. Maeda et al. have purified rice endo- $\beta-N$ acetylglucosaminidase and show that the substrate specificity of the native and recombinant enzyme are almost the same. The same group reports that oligomannosidic free $N$-glycans are present in microsomes isolated from pumpkin hypocotyls which leads to the interesting model that oligomannosidic free $N$-glycans could be taken up by the ER, from where they are secreted through the Golgi to the extracellular space (Katsube et al.). Like glycoproteins, the free $\mathrm{N}$-glycans are processed in the Golgi, but the biological relevance of secreted complex-type free $N$-glycans is unknown.

Tobacco BY-2 cell suspensions are an established model system for plant cell biology (Nagata et al., 1992) and used as production platform for recombinant proteins (Santos et al., 2016). Herman et al. use CRISPR/Cas9 genome editing to produce homogenous mannosidic $N$-glycans on BY-2 cell produced glycoproteins. Intriguingly, the loss-of-function approach reveals a new aspect of the $N$-glycan processing pathway. Knockout of $\mathrm{N}$-acetylglucosaminyl transferase I (GNTI), the key enzyme for complex $N$-glycan formation, is not sufficient to eliminate all $\mathrm{N}$-glycans with core fucose suggesting that core fucosylation is not strictly dependent on the GlcNAc residue transferred by GNTI. In tomato, GNTI and complex $\mathrm{N}$-glycans are crucial for development. Knockdown of GNTI in tomato causes necrotic fruit-attached stalks, early fruit drop, and patchy fruit ripening (Kaulfürst-Soboll et al.). By contrast, only subtle phenotypes are observed when hybrid $N$-glycans are generated by silencing of Golgi $\alpha$-mannosidase II, which catalyzes the subsequent processing reaction in the pathway.

Using a label-free quantitative proteomics approach Liu, Niu et al. identify proteins that are differentially regulated under salt stress in Arabidopsis thaliana mutants lacking complex Nglycans and uncover several glycoproteins that could be involved in salt stress tolerance. Frank et al. examine the effect of synthetic phytohormones on root hair formation in Arabidopsis mutants with blocked or altered complex $N$-glycan processing. The lack of complex $\mathrm{N}$-glycans in the gntI mutant causes increased root hair elongation and hypersensitivity to a synthetic auxin when compared to wild-type roots. Lewis A containing structures are the most elaborate complex-type $N$-glycans found in plants (Strasser et al.). Strikingly, Arabidopsis mutants with impaired biosynthesis of Lewis A structures display also elongated root hairs demonstrating for the first time a biological role of Lewis A containing $\mathrm{N}$-glycans (Frank et al.). In Arabidopsis, the occurrence of Lewis A containing glycoproteins is tissuespecific with high amounts in stems and siliques and low levels in roots (Strasser et al., 2007). Beihammer, Maresch, Altmann, Van Damme et al. use the Lewis A specific JIM84 antibody to identify by affinity purification and nano-LC-MS analysis Lewis A containing glycoproteins from Arabidopsis, rice and Nicotiana benthamiana and show that they may be associated with different functions in different plant species. By this approach they also demonstrate that the Lewis A structures are highly conserved in different Arabidopsis accessions. Using a similar approach, the identification of Lewis A-containing glycoproteins from root hairs may in the future pave the way to reveal the mechanisms underlying the altered auxin sensitivity and the role of complex $\mathrm{N}$-glycans in the regulation of root hair elongation.

While the formation of Lewis A type structures takes place in the trans-Golgi, truncated or paucimannosidic $N$-glycans are generated in a downstream compartment or in the apoplast by removal of one or two terminal GlcNAc residues. Alvisi et al. report that the three $N$. benthamiana $\beta$-hexosaminidases (HEXO1-HEXO3) have distinct substrate specificities. HEXO1 and HEXO3 cleave off GlcNAc residues from complex $\mathrm{N}$ glycans and both, HEXO2 and HEXO3, are capable of GalNAc trimming from $N$-glycans. Plant $N$-glycans are normally not decorated with GalNAc residues, but the study has important implications for glycoengineering approaches aiming at the generation of homogenous $\mathrm{N}$ - and $\mathrm{O}$-linked glycan structures found in helminths or mammals (Castilho et al., 2012; Wilbers et al., 2017).

Compared to higher plants, little is still known about the biosynthesis of $\mathrm{N}$ - and $\mathrm{O}$-glycans in microalgae which display a remarkable structural diversity. Recent advances in characterization of $\mathrm{N}$-glycans isolated from different microalgae species are nicely summarized in the review by Mathieu-Rivet et al. Differences in $N$-glycan precursor biosynthesis and processing steps in the Golgi result in an unexpected variety of $N$-glycan structures that appear characteristic for microalgae. Mócsai, Kaehlig et al. add another facet to the complexity of major $N$-glycans found in different microalgae. Using a combination of independent techniques including MALDI-TOF-MS, linkage analysis by GC-MS and NMR, the authors reveal the structural differences of uncommon isobaric $N$-glycan structures that are derived from phylogenetically divergent microalgae species.

\section{O-GLYCOSYLATION: ARABINOGALACTAN PROTEINS, HYDROXYPROLINE-RICH GLYCOPROTEINS AND CELL WALL POLYMERS}

Another notable aspect of this Research Topic is the interest in protein $O$-glycosylation and hydroxyproline-rich glycoproteins (HRGPs) in the cell wall. An impressive six articles review protein glycosylation in plants, summarizing our present knowledge of both $\mathrm{N}$ - and $\mathrm{O}$-glycosylation in higher plants (Strasser et al.) and microalgae (Mathieu-Rivet et al.), as well as highlighting structural-functional aspects of arabinogalactan-proteins (AGPs) (Seifert, Silva et al.) and of extensins, with emphasis on arabinosylation (Petersen et al.) and oxidative crosslinking (Mishler-Elmore et al.), respectively.

Most O-glycans in plants, including microalgae (Mathieu-Rivet et al.) are attached to hydroxyproline (Hyp), an amino acid that is generated post-translationally by the action of prolyl-4-hydroxylases $(\mathrm{P} 4 \mathrm{H})$, as reported in two research articles in this Research Topic. Mócsai, Göritzer et al. take a long overdue new attempt at the substrate specificity of $\mathrm{P} 4 \mathrm{H}$ isoforms, albeit with the result that four individual $\mathrm{N}$. benthamiana $\mathrm{P} 4 \mathrm{H}$ isoforms do not show differences in substrate selectivity in a non-plant expression system. In contrast, the 
study by Konkina et al. demonstrates a specific role for the A. thaliana P4H3 gene in AGP abundance and tolerance to hypoxia. Hence, the mechanism of $\mathrm{P} 4 \mathrm{H}$ selectivity remains an open question.

The build-up of Hyp-linked $O$-glycan structures requires a multitude of glycosyl transferases several of which are presently known (Strasser et al.; Petersen et al.; Silva et al.). In this issue, the A. thaliana GALT8 locus is described to encode an enzyme with $\beta-(1,3)$ galactosyl transferase activity in vitro (Narciso et al.). This activity is needed for the elaboration of arabinogalactan (AG) type II structures found on all AGPs and some pectic polymers. Thus, GALT8's biochemical function is consistent with the global growth phenotype of galt8 single mutants. However, the weakness of the galt8 phenotype might be due to partial redundancy with the KNS4 and the Atlg77810 $\beta-(1,3)$ galactosyl transferase encoding loci. Type II AG on some AGPs contains $\alpha-(1,2)$ linked terminal fucose and it is believed that the Arabidopsis FUT4 and FUT6 loci encode the corresponding fucosyl transferases (Strasser et al.; Silva et al.). New biochemical in vitro work presented in this Research Topic in principle widens the activities of FUT4 and FUT6 to any $\alpha-(1,3)$ and $\alpha-(1,5)$ linked terminal arabinofuranose which includes type II AG but also non-AGP like oligosaccharides (Soto et al.).

One subfamily of AGP is defined by their fasciclin1 domains that have been hypothesized to interact with proteins or carbohydrates. These FASCICLIN LIKE AGPs (FLAs) are known to act in different developmental roles and two research articles demonstrate non-redundant roles for two A. thaliana FLA loci encoding group B glycoproteins that in contrast to most FLAs do not contain GPI-anchors. The study by Liu, MacMillan et al. shows that FLA16 expression correlates with secondary cell wall formation and that the FLA16 protein is a moderately glycosylated protein localized to the plasma membrane and the apoplast. Intriguingly, the fla16 loss of function mutation lacks normal stem strength and cellulose content indicating a role of FLA16 in secondary cellulose formation similar to what has previously been described for FLA11 and -12 (MacMillan et al., 2010). Another group B FLA locus called FLA18 is the subject of the paper by Allelign Ashagre et al.. In contrast to the secondary growth-related FLA16, FLA18 expression correlates with expansion growth in roots i.e. primary cell wall formation. The root swelling fla18 phenotype that depends on sucrose-levels in the growth medium is reminiscent of the fla4 mutant but specific to lateral roots. However, the genetic interaction between the loci is synergistic suggesting independent roles of the two FLAs in the process of root elongation. Despite these intriguing first descriptions of single loss of function phenotypes of group B fla mutants, the mode of action of the entire FLA family remains largely obscure.

Finally, two research papers present genome-wide surveys of HRGPs (Abedi et al.) and potentially glycan binding, lectindomain proteins (Petrova et al.) in relation to cell wall formation in the fiber crops poplar and flax, respectively. Both studies conclude that expression of many cell wall proteins is closely correlated with the formation of polysaccharides of different cell wall types, which is also in line with the two aforementioned functional studies on FLA16 and FLA18.
In contrast to the raft of papers on cell wall proteins, relatively few contributions in this Research Topic deal with cell wall polysaccharide biosynthesis. The review by Zabotina et al. surveys the emerging theme of complex formation in the biosynthesis of cell wall matrix polymers in the Golgi. While this field is experimentally challenging it opens up the possibility of regulating polysaccharide abundance and structure at the cellular level and is of equal importance as the cellulose synthase complex formation and motility to fully understand cell wall biosynthesis. However, also the search for new components in cell wall polysaccharide biosynthesis is far from over as exemplified by the knock-out study of the only orthologous gene to pectic rhamnogalacturonan I (RG I) backbone synthesizing rhamnosyl transferase (RRT) in liverwort Marchantia polymorpha, published in this Research Topic (Wachananawat et al.). While MpRRT1 should be essential for RG I biosynthesis the mprrt1 mutant still contains $80 \%$ of the normal RG I content pointing to a contribution of other RG I backbone synthesizing loci that remain to be identified.

\section{PROTEIN-CARBOHYDRATE INTERACTIONS, UP TO APPLICATIONS}

The specific interaction between proteins such as carbohydrateactive enzymes and lectins, and carbohydrate structures is at the forefront plant glycobiology. Consequently, several papers in this Research Topic aim at the (functional) characterization of glycosyl transferases, lectins, GPI anchor modifications and their importance for plant development.

Beihammer, Maresch, Altmann et al. provide insights into the biosynthesis of the lipid-linked glycan backbone that is subsequently transferred to proteins during the attachment of a GPI anchor. MS analysis and glycosidase digestion show that the core glycan of a GPI-anchor derived from a reporter protein carries an additional galactose residue that is likely transferred in the Golgi.

Romero-Pérez et al. report in their study that overexpression of the chimeric lectin F-box Nictaba helps Arabidopsis plants to cope with bacterial infection from Pseudomonas syringae. In addition, higher levels of anthocyanins are accumulating in overexpression lines compared to wild-type plants and knockout lines.

Putative lectins are the subject of a bioinformatic transcriptome analysis of gene expression in various flax tissues producing different types of cell walls. From this analysis it is predicted that the multiple lectin mediated processes take place in plant cell walls throughout plant development (Petrova et al.).

Functional studies are an important step toward possible applications of glycobiology research. Zhang and Showalter give an overview of the principles and use of CRISPR/Cas9 to perform functional analysis of genes important for plant cell wall biosynthesis. While the application of genome editing tools to questions related to cell wall biology is still limited to a few examples, the authors show that because of the high specificity and the possibility for multi-gene targeting CRISPR/Cas9 is 
an ideal tool to uncover biological functions of genes, which can be extended and applied to many other genes encoding carbohydrate-modifying enzymes, and beyond. Shin et al. used the $N$. benthamiana platform for functional expression of the SARS-CoV-2 receptor binding domain. Though $N$-glycosylation of the receptor binding domain is important for proper folding, the $N$-glycan processing did not affect its functionality.

In the past decades, plants have become a very attractive platform for recombinant protein production of glycoproteins. Consequently, several model plants have been glyco-engineered to enable synthesis of human $\mathrm{N}$-glycan structures in plants (Montero-Morales and Steinkellner, 2018; Margolin et al., 2020). Bohlender et al. were successful in establishing stable protein sialylation in Physcomitrella patens, creating opportunities for this moss to be used as a plant-based biopharmaceutical production platform. In their review paper Margolin et al. highlight recent progress in glycoengineering and the engineering of glycosylation-directed folding pathways in plants, aiming for use in the production of recombinant viral glycoprotein vaccines (Gitzinger et al., 2009; Castilho et al., 2010).

Although many papers in this Research Topic still focus on A. thaliana as a model plant, several other models, such as microalgae, $P$. patens, $M$. polymorpha, N. benthamiana, and $N$.

\section{REFERENCES}

Castilho, A., Neumann, L., Daskalova, S., Mason, H. S., Steinkellner, H., Altmann, F., et al. (2012). Engineering of sialylated mucin-type O-glycosylation in plants. J. Biol. Chem. 287, 36518-36526. doi: 10.1074/jbc.M112.402685

Castilho, A., Strasser, R., Stadlmann, J., Grass, J., Jez, J., Gattinger, P., et al. (2010). In planta protein sialylation through overexpression of the respective mammalian pathway. J. Biol. Chem. 285, 15923-15930. doi: 10.1074/jbc.M109.088401

De Coninck, T., Gistelinck, K., Janse van Rensburg, H.C., Van den Ende, W., and Van Damme, E.J.M. (2021). Sweet modifications modulate plant development. Biomolecules 11:756. doi: 10.3390/biom11050756

Gitzinger, M., Parsons, J., Reski, R., and Fussenegger, M. (2009). Functional cross-kingdom conservation of mammalian and moss (Physcomitrella patens) transcription, translation and secretion machineries. Plant Biotechnol. J. 7, 73-86. doi: 10.1111/j.1467-7652.2008.00376.x

MacMillan, C.P., Mansfield, S.D., Stachurski, Z.H., Evans, R., and Southerton, S.G. (2010). Fasciclin-like arabinogalactan proteins: specialization for stem biomechanics and cell wall architecture in Arabidopsis and Eucalyptus. Plant J. 62, 689-703. doi: 10.1111/j.1365-313X.2010.04181.x

Margolin, E.A., Strasser, R., Chapman, R., Williamson, A.L., Rybicki, E.P., and Meyers, A.E. (2020). Engineering the plant secretory pathway for the production of next-generation pharmaceuticals. Trends Biotechnol. 38, 1034-1044. doi: 10.1016/j.tibtech.2020.03.004

Montero-Morales, L., and Steinkellner, H. (2018). Advanced plant-based glycan engineering. Front. Bioeng. Biotechnol. 6:81. doi: 10.3389/fbioe.2018.00081

Nagashima, Y., von Schaewen, A., and Koiwa, H. (2018). Function of N-glycosylation in plants. Plant Sci. 274, 70-79. doi: 10.1016/j.plantsci.2018.05.007

Nagata, T., Nemoto, Y., and Hasezawa, S. (1992). Tobacco BY-2 cell line as the "HeLa" cell in the cell biology of higher plants. Int. Rev. Cytology 132, 1-30. doi: 10.1016/S0074-7696(08)62452-3 tabacum, Populus, flax, tomato, and rice are now also being used in plant glycobiology studies. Clearly this Research Topic highlights the progress made in the field of plant glycobiology, and emphasizes the importance of glycosylated molecules and polysaccharides for plant growth and development.

\section{AUTHOR CONTRIBUTIONS}

All authors contributed equally to this manuscript and approved the final version.

\section{FUNDING}

This work was supported by the Austrian Science Fund (FWF) Project P31920-B32 and P32332-B and the Research Foundation-Flanders (FWO Project G002416N).

\section{ACKNOWLEDGMENTS}

We would like to thank all authors for their contributions to this Research Topic. Furthermore, we are grateful to all reviewers and editors for their help in evaluating all manuscripts and the editorial office for their support.
Santos, R.B., Abranches, R., Fischer, R., Sack, M., and Holland, T. (2016). Putting the spotlight back on plant suspension cultures. Front. Plant Sci. 7:297. doi: 10.3389/fpls.2016.00297

Strasser, R. (2016). Plant protein glycosylation. Glycobiology 26, 926-939. doi: 10.1093/glycob/cww023

Strasser, R., Bondili, J. S., Vavra, U., Schoberer, J., Svoboda, B., Glössl, J., et al. (2007). A unique beta1,3-galactosyltransferase is indispensable for the biosynthesis of N-glycans containing Lewis A structures in Arabidopsis thaliana. Plant Cell 19, 2278-2292. doi: 10.1105/tpc.107.052985

Wilbers, R.H.P., Westerhof, L.B., Van Noort, K., Obieglo, K., Driessen, N.N., Everts, B., et al. (2017). Production and glyco-engineering of immunomodulatory helminth glycoproteins in plants. Sci. Rep. 7, 1-10. doi: 10.1038/srep45910

Conflict of Interest: The authors declare that the research was conducted in the absence of any commercial or financial relationships that could be construed as a potential conflict of interest.

Publisher's Note: All claims expressed in this article are solely those of the authors and do not necessarily represent those of their affiliated organizations, or those of the publisher, the editors and the reviewers. Any product that may be evaluated in this article, or claim that may be made by its manufacturer, is not guaranteed or endorsed by the publisher.

Copyright (c) 2021 Seifert, Strasser and Van Damme. This is an open-access article distributed under the terms of the Creative Commons Attribution License (CC BY). The use, distribution or reproduction in other forums is permitted, provided the original author(s) and the copyright owner(s) are credited and that the original publication in this journal is cited, in accordance with accepted academic practice. No use, distribution or reproduction is permitted which does not comply with these terms. 